\title{
Effects of hydroxyapatite extract on rats with transient ischemia: Long-term potentiation and axon regeneration
}

\author{
JING XU ${ }^{1}$, CHUNYANG WANG ${ }^{1}$ and PENGJUAN XU ${ }^{2}$ \\ ${ }^{1}$ Department of Neurology, Tianjin Medical University General Hospital, Tianjin 300052; ${ }^{2}$ School of Integrative Medicine, \\ Tianjin University of Traditional Chinese Medicine, Tianjin 301617, P.R. China
}

Received April 3, 2020; Accepted August 10, 2021

DOI: $10.3892 /$ etm.2021.10921

\begin{abstract}
Hydroxyapatite (HA) has been extensively used as a reconstructive and prosthetic material for osseous tissue. The present study aimed to determine whether HA extract exerted effects on central nervous system injury following transient cerebral ischemia/reperfusion in rats. Male Wistar rats were treated with $\mathrm{HA}$ following bilateral common carotid artery clamping (two-vessel occlusion). The results demonstrated that treatment with HA extract attenuated the inhibition of long-term potential in a rat model of transient cerebral ischemia/reperfusion. Furthermore, HA extract improved axon regeneration, which was confirmed via the immunohistochemical analysis of growth associated protein 43 and glial fibrillary acidic protein. Taken together, the results of the present study provided preliminary evidence of the protective effect of HA on neuronal damage.
\end{abstract}

\section{Introduction}

Cerebral ischemia is one of the major leading causes of morbidity and mortality worldwide (1). Axonal regeneration occurs in the central nervous system (CNS) following cerebral ischemia (2). The re-establishment of appropriate synaptic networks following axonal sprouting is necessary to restore cognitive function following ischemia injury (3). Thus, current treatment strategies target axonal regeneration following CNS injury (4).

Hydroxyapatite (HA) is a natural component that is responsible for the strength and stability of the human skeletal system (5). Owing to its notable biocompatibility and bioactivity, HA has been extensively used as reconstructive and prosthetic material for the repair and regeneration of osseous

Correspondence to: Dr Jing Xu, Department of Neurology, Tianjin Medical University General Hospital, 154 Anshan Road, Heping, Tianjin 300052, P.R. China

E-mail: jingxu01@tmu.edu.cn

Key words: hydroxyapatite, ischemia, long term potential, growth associated protein 43 , glial fibrillary acidic protein tissue (6). Ion exchange takes place between HA and body fluid following HA implantation into the bone. In addition, HA releases calcium and phosphorus to its surroundings, which along with other molecules, enters the blood circulation and effects the CNS (5). To the best of our knowledge, there is limited evidence denoting the effect of HA extract on the CNS.

$\mathrm{HA}$ is non-toxic to various cells, including human monocyte-derived macrophages (7), human osteosarcoma osteoblasts (8) and mesenchymal stem cells (8). In addition, a previous study has demonstrated the ability of HA to promote the attachment of human osteoblast-like cells (7) to improve the adhesion and proliferation of both human osteosarcoma osteoblasts (MG63 cells) and mesenchymal stem cells (8). HA exerts notable bioactivity, suggesting that it interacts with cells and tissues, stimulating their repair and regeneration (5). Thus, the present study hypothesized that HA may improve the growth of injured neurons in conditions of axonal sprouting following CNS injury.

A transient ischemia/reperfusion rat model, involving bilateral common carotid artery clamping, can decrease blood flow in the brain to one-third of its normal value (9). CNS injury also affects the hippocampus (10), which is associated with several neuronal properties, including neuronal cell viability or the electrophysiological behavior of neurons (11). The most-studied cellular model for hippocampal learning and memory is the detection of long term potential (LTP), which is a long lasting increase in the synaptic transmission efficiency induced by high frequency stimulation (12).

Ischemic brain insults, whether caused by global or focal cerebral ischemia, may induce neurogenesis (13). Growth associated protein 43 (GAP43) is a membrane-bound protein that is located in the axonal growth cones of sprouting CNS axons (14); it has been extensively used to quantitate sprouting axons during neuroanatomical remodeling (15). Previous evidence has demonstrated that the association between astrocytes and neurons is important for protecting the CNS against different types of insult, including cerebral ischemia and hypoxia, or neurological disorders $(16,17)$. Glial fibrillary acidic protein (GFAP) is the major cytoskeletal protein of astrocytes in the brain (18). Previous study reported that GFAP expression is upregulated in different types of brain injury, including trauma, demyelination and brain ischemia (18). Thus, GFAP was used as a marker for the histological examination of brain tissue in the present study. 
The present study aimed to determine whether HA extracts reverse the LTP deficit and improve axon regeneration in a rat model of ischemia/reperfusion. The results of the present study provide novel insights into LTP and the histological changes of brain tissues, based on GFAP and GAP43 expression levels following transient cerebral ischemia/reperfusion.

\section{Materials and methods}

Preparation of HA extracts. Wet precipitation was performed to prepare $\mathrm{HA}$ with a $\mathrm{Ca} / \mathrm{P}$ ratio of 1.67 (7). Aqueous extracts from HA (a gift from Professor Jie Huang, University College of London) were prepared by adding $5 \mathrm{~g} \mathrm{HA}$ in $100 \mathrm{ml}$ physiological saline at $37^{\circ} \mathrm{C}$ for $72 \mathrm{~h}$. All extracts were sterilized via filtration through a $0.20 \mathrm{~mm}$ filter and the collected solution was stored at $4^{\circ} \mathrm{C}$ for $12 \mathrm{~h}$ for intraperitoneal injection.

Animal model and drug administration. Male Wistar rats (purchased from the Laboratory Animal Center of Academy of Military Medical Science of People's Liberation Army, China; 6 weeks old; 270-300 g; $\mathrm{n}=24$ ) were randomly divided into three groups ( $n=8$ per group): The sham-operated group, the bilateral common carotid artery occlusion (2-VO) group and the HA-treated group. Animals were housed with free access to water and food under a $12 \mathrm{~h}$ light/dark cycle in a $22-25^{\circ} \mathrm{C}$ with 40-60\% humidity. The animal care and experimental protocols were approved by the Medical Ethics Committee of Tianjin Medical University General Hospital (Tianjin, China).

In the sham-operated group, the carotid arteries were exposed but not occluded. Rats were subjected to transient cerebral ischemia/reperfusion in both the 2-VO and HA-treated groups. Prior to induction of transient cerebral ischemia, rats were anaesthetized with $10 \%$ chloralhydrate (intraperitoneal, $300 \mathrm{mg} / \mathrm{kg}$ ), and exhibited no signs of peritonitis following administration. The common carotid arteries were isolated and clamped using non-traumatic aneurysm clips for $30 \mathrm{~min}$. The clips were subsequently released for $10 \mathrm{~min}$ and a second ischemia was applied for $30 \mathrm{~min}$.

On day 1 after surgery, rats of the HA-treated group were administered HA saline solution via intraperitoneal injection at $18 \mathrm{mg} / 100 \mathrm{~g}$ body weight dissolved in $1 \mathrm{ml}$ PBS per day. This regimen was performed for a total of 14 days. In the sham and 2-VO groups, rats were injected with an equal volume of PBS.

Electrophysiological recordings. Electrophysiological recordings were obtained at 14 days post-surgery. Rats were anesthetized with urethane $(1 \mathrm{~g} / \mathrm{kg})$ and subsequently placed in a stereotaxic frame (Narishige International, Ltd.). Small holes were drilled into the skull to insert stimulatory and recording electrodes (A.M.P.I., Jerusalem, Israel). The tip of the recording electrode was positioned in the stratum radiatum of the cornu ammonis region 1 (CA1) area $(3.5 \mathrm{~mm}$ posterior to the bregma and $2.5 \mathrm{~mm}$ lateral to the midline). The stimulating electrode was inserted into the CA3 region $(4.2 \mathrm{~mm}$ posterior to the bregma and $3.5 \mathrm{~mm}$ to the midline). The test stimuli (0.3-0.5 mA) were delivered to the CA3 region every $30 \mathrm{sec}$ at an intensity that evoked a response of $50 \%$ of its maximum. After recording every $20 \mathrm{sec}$ for $20 \mathrm{~min}$ to produce stable baseline data, a high frequency stimulation consisting of 10 trains of 10 stimuli at $100 \mathrm{~Hz}$ with $2 \mathrm{sec}$ intertrain intervals was delivered to induce LTP. The field excitatory postsynaptic potential (fEPSP)was subsequently recorded at $40 \mathrm{kHz}$ every $20 \mathrm{sec}$ for $1 \mathrm{~h}$ using Scope software (PowerLab; AD Instruments). Finally, the fEPSP slope was normalized and analyzed using Clampfifit 9.0 (Molecular Devices, LLC).

Histology and immunohistochemistry (IHC) analyses. Following electrophysiological analysis, the rats were deeply anesthetized with urethane $(1 \mathrm{~g} / \mathrm{kg})$ and perfused through the left cardiac ventricle with PBS (pH 7.2), followed by $4 \%$ paraformaldehyde in $0.1 \mathrm{M}$ phosphate buffer ( $\mathrm{pH}$ 7.2). The brain of each rat was removed, fixed by immersion in the same solution and embedded in paraffin. Paraffin-embedded tissue sections were then cut into 5- $\mu \mathrm{m}$-thick sections. Tissue sections were placed onto uncoated slides prior to hematoxylin and eosin (H\&E)staining, while sections intended for IHC analysis were placed onto coated slides (ZSJQ-BIO). H\&E staining using light microscopy was routinely performed for the assessment of histomorphology.

Immunohistochemical procedures for GFAP and GAP-43. Tissue sections intended for immunoreactivity analysis were placed onto coated slides (OriGene Technologies, Inc.). Labeled dextran polymer IHC was used to detect the expression levels of GAP43 and GFAP in CA1 subfields. Deparaffinized tissue sections were boiled in citrate buffer for antigen retrieval and incubated with $3 \% \mathrm{H}_{2} \mathrm{O}_{2}$ solution for $30 \mathrm{~min}$ at room temperature to inhibit endogenous peroxidase activity. Tissue sections were incubated with rabbit polyclonal anti-GFAP (1:100; ZSJQ-BIO) or rabbit polyclonal anti-GFAP (1:100; Newmarker Biotechnology) antibodies overnight at $4^{\circ} \mathrm{C}$. PBS replaced the antibodies as the negative controls. Following the primary incubation, tissue sections were incubated with En Vision-Systems polymer-conjugated PV-9000 secondary antibody (GBI), and subsequently incubated with $\mathrm{DAB}$ at room temperature for $3 \mathrm{~min}$. Between steps, the slides were washed twice with PBS. Tissue sections were observed and imaged using an Olympus YS100 microscope (Olympus Corporation) with a CCD camera (JVC).

Statistical analysis. SPSS software (version 16.0; SPSS, Inc.) was used for statistical analysis. Data are presented as the mean \pm standard error of the mean. One-way ANOVA followed by Bonferroni's post hoc test was used to analyze the data. $\mathrm{P}<0.05$ was considered to indicate a statistically significant difference.

\section{Results}

Change of LTP. The mean normalized slope of the fEPSP across the 60-min assessment period for each animal was significantly decreased in the 2-VO group compared with that in the sham-operated group (2-VO, 100.9 $3.4 \%$; sham-operated, 133.4+3.3\%; $\mathrm{P}<0.05$; Fig. 1). Notably, this change was partially recovered following treatment with $\mathrm{HA}$ (HA, 105.2 $\pm 3.1 \%$; $\mathrm{P}<0.05$ vs. sham or 2-VO; Fig. 1).

$H \& E$ staining. In the hippocampus, marked morphological changes were detected in the ischemic group, including neuronal cell loss, glial proliferation, nuclei shrinkage, cere- 


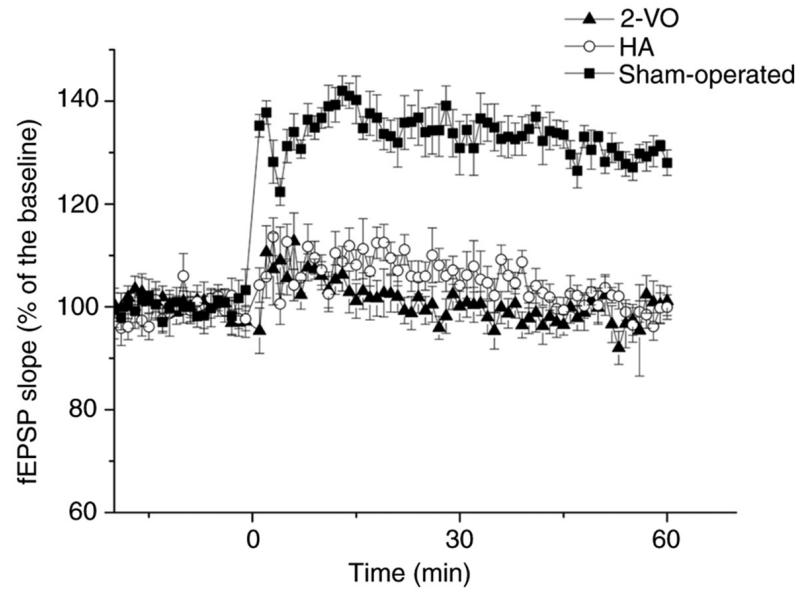

Figure 1. Effects of HA on the long-term potential of the fEPSP slope in the three groups. Data are presented as the mean \pm standard error of the mean. HA, hydroxyapatite; 2-VO, bilateral common carotid artery occlusion; fEPSP, field excitatory postsynaptic potential.
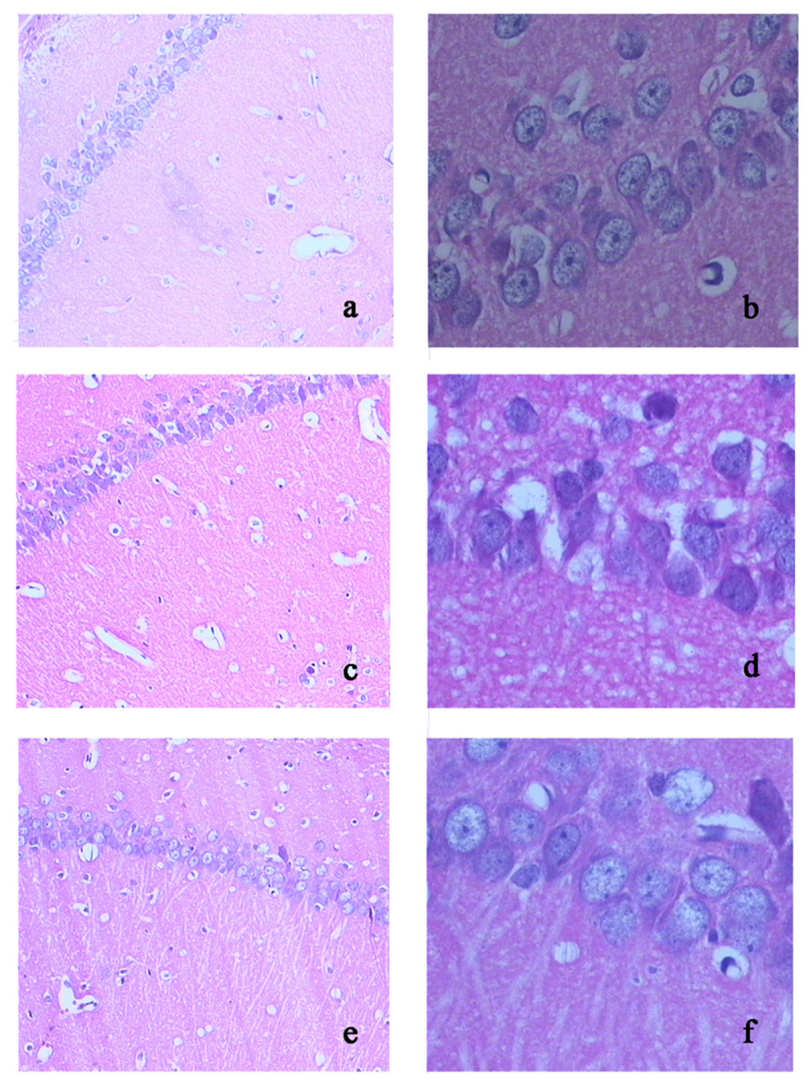

Figure 2. Effects of HA on the morphological changes of rat hippocampi induced by transient cerebral ischemia/reperfusion. Images of hematoxylin and eosin staining were obtained at (a) x100 and (b) x400 magnification in the sham-operated group, (c) x100 and (d) x400 magnification in the bilateral common carotid artery occlusion group and (e) x100 and (f) x400 magnification in the HA-treated group. HA, hydroxyapatite.

bral edema and dark staining of neurons, all of which were observed in the hippocampal CA1 region. Treatment with HA markedly decreased these pathological changes (Fig. 2a and f).

IHC analysis of GAP43 and GFAP. To investigate the effect of HA on axonal growth following transient cerebral isch-
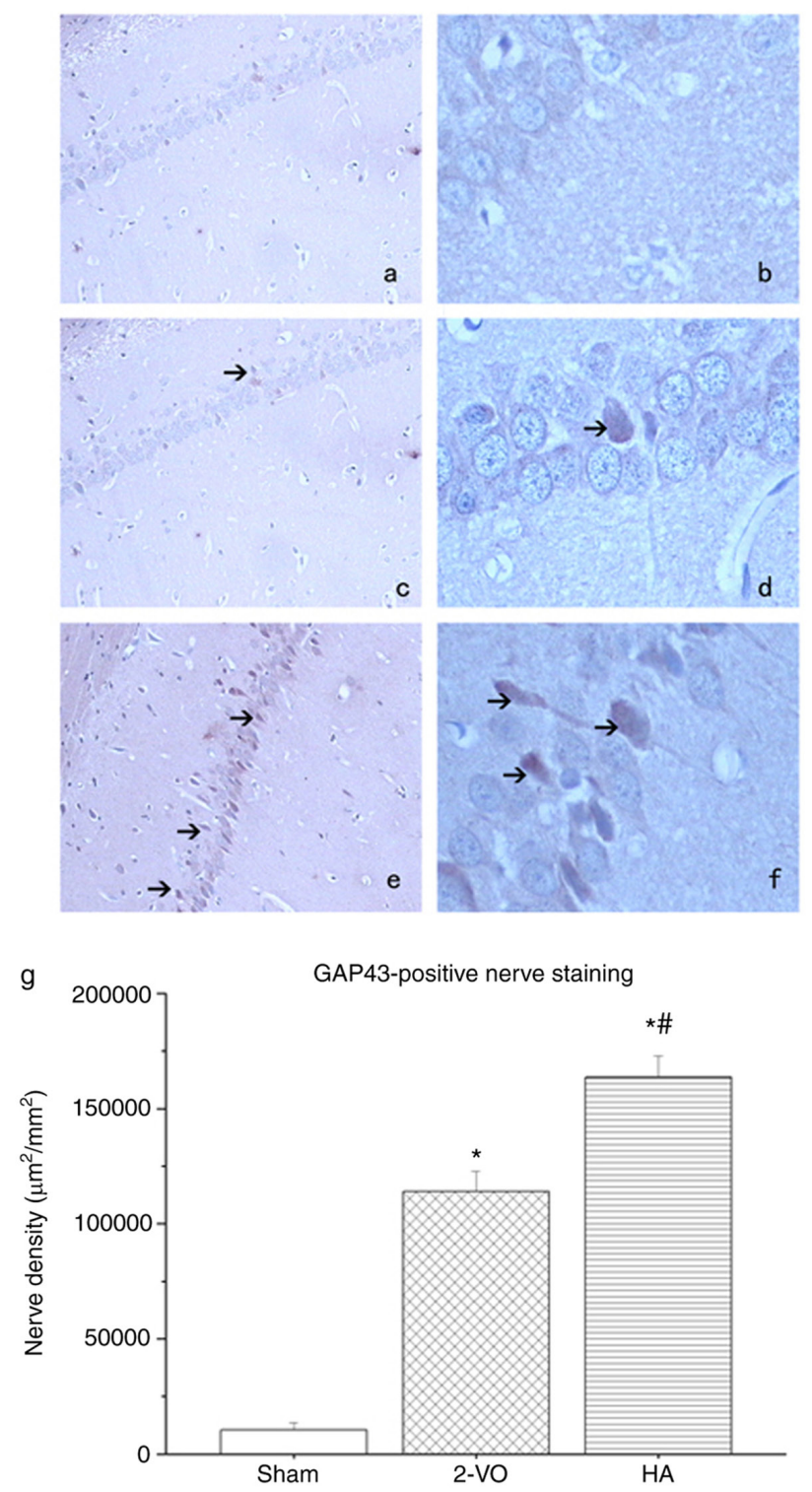

Figure 3. GAP43 expression following transient cerebral ischemia/reperfusion. Images of the sham-operated group at (a) $\times 100$ and (b) $\times 400$ magnification were obtained. The 2-VO group was assessed at (c) x100 and (d) x400 magnification, and the HA-treated group was also assessed at (e) x100 and (f) $\times 400$ magnification. (g) GAP43-positive nerve staining. Data are presented as the mean \pm standard error. ${ }^{*} \mathrm{P}<0.05$ vs. control, ${ }^{~} \mathrm{P}<0.05$ vs. 2 -VO. Black arrows indicate positive GAP43 signals. GAP43, growth associated protein-43; 2-VO, bilateral common carotid artery occlusion; HA, hydroxyapatite.

emia/reperfusion, the expression levels of GAP43 and GFAP in the hippocampus of rat brains were compared. As presented in Fig. 3, GAP43 had more prominent localization in the HA-treated group compared with that in the $2-\mathrm{VO}$ group $(163,596 \pm 9,263$ vs. $113,892 \pm 8,972 \mathrm{~m}^{2} / \mathrm{mm}^{2} ; \mathrm{P}<0.05$ ). Additionally, when compared with the 2-VO and HA groups, the lowest expression of GAP43 was observed in the sham-operated group $\left(10,364 \pm 3,298 \mathrm{~m}^{2} / \mathrm{mm}^{2} ; \mathrm{P}<0.05\right.$ in the 2 -VO and HA groups vs. the sham-operated group). As presented in Fig. 4, lower GFAP positive signals were expressed in the hippocampus of the HA-treated group compared with those in the 2-VO group $(29.73 \pm 3.28$ vs. $70.95 \pm 5.68 ; \mathrm{P}<0.05)$, with the lowest expression in the sham-operated group $(10.5 \pm 1.03 ; \mathrm{P}<0.05$ in the $2-\mathrm{VO}$ and HA groups vs. the sham-operated group). 

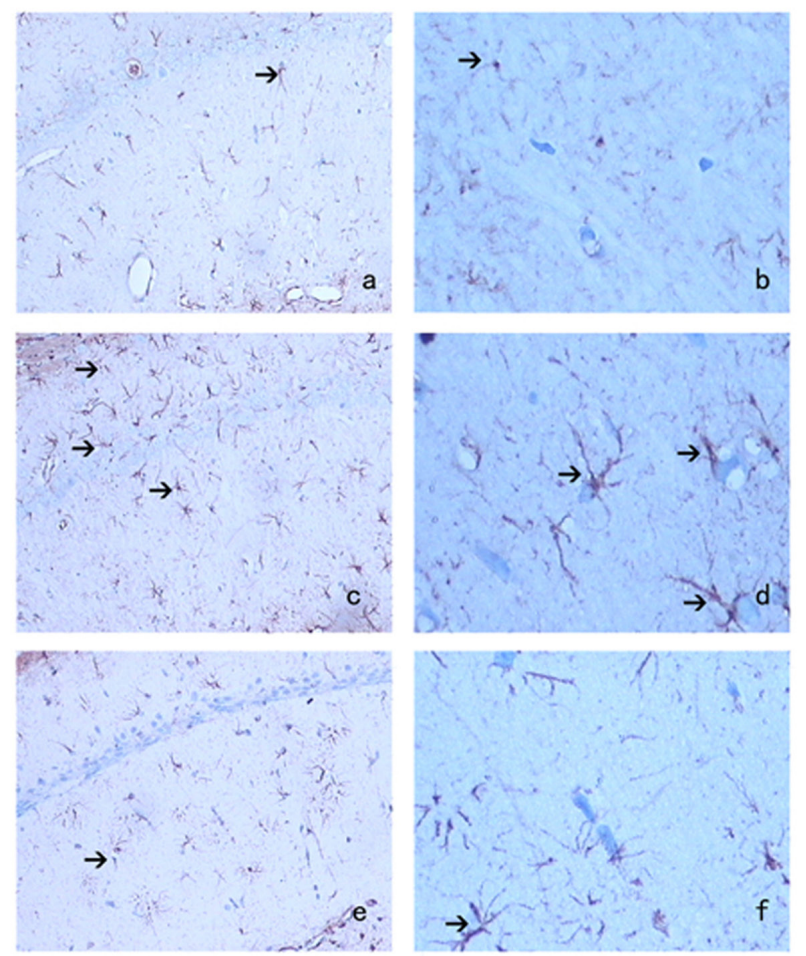

g

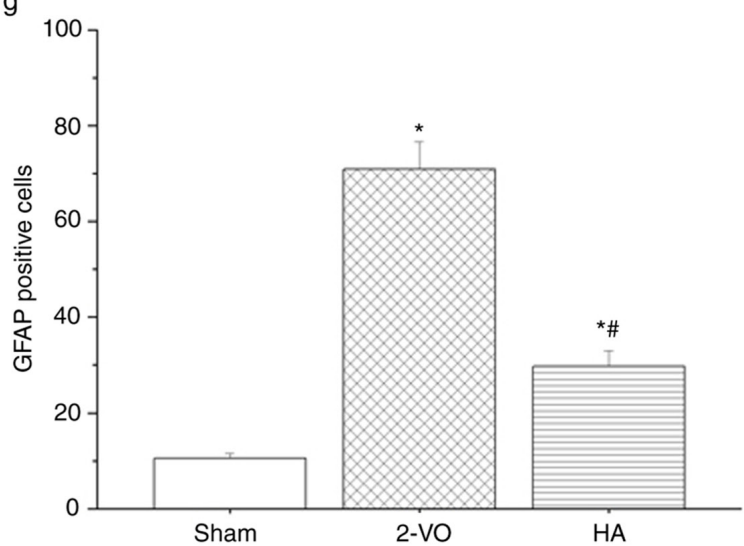

Figure 4. GFAP expression following transient cerebral ischemia/reperfusion. Images of the sham-operated group at (a) x100 and (b) x400 magnification were obtained. The 2-VO group was assessed at (c) x100 and (d) x400 magnification, and the HA-treated group was also assessed at (e) x100 and (f) $x 400$ magnification. (g) GFAP positive cells. Data are presented as the mean \pm standard error. ${ }^{~} \mathrm{P}<0.05$ vs. control, ${ }^{\text {}} \mathrm{P}<0.05$ vs. 2 -VO. Black arrows represent positive GFAP signals. GFAP, glial fibrillary acidic protein; 2-VO, bilateral common carotid artery occlusion; HA, hydroxyapatite.

\section{Discussion}

Bilateral common carotid artery clamping decreases blood flow in the brain to one-third of its normal value in rats (9). Given that hypoperfusion also affects the hippocampus (10), it may exert effects on several neuronal properties, including neuronal cell viability or the electrophysiological behavior of neurons (11). Thus, LTP impairment may be expected to occur in rat hippocampal Schaffer collateral-CA1 synapses.

The results of the present study demonstrated that LTP was inhibited in both the HA-treated and 2-VO groups following transient cerebral ischemia/reperfusion when compared with the sham-operated group. These results confirmed the utility of this model in the investigation of HA effects. The reconstruction of synapses is essential in developing LTP following injury (19), and the results of the present study demonstrated that administration of HA extracts decreases transient cerebral ischemia/reperfusion-induced LTP impairment, suggesting that HA extracts may be used to improve the reconstruction of synapses following transient cerebral ischemia/reperfusion.

A previous study demonstrated that a reduction in cerebral blood flow, and the concomitant abnormalities of energy metabolism that arise in chronic cerebral ischemia can lead to the selective neuronal injuries in vulnerable regions of the brain, particularly the hippocampus and cerebral cortex (20). The results of the present study were consistent with these findings, as H\&E staining exhibited neuronal cell loss, glial proliferation, nuclei shrinkage, cerebral edema and dark staining of neurons in the hippocampus of both the HA-treated and 2-VO groups following transient cerebral ischemia/reperfusion. Treatment with HA extracts markedly decreased these pathological changes, which initially indicated the neuroprotective effects of HA extracts on the CNS following ischemia.

GAP43 was selected as an indicator of axonal regeneration in the present study. Neurons can extend axon branches and form new connections following injury (21). It is also known that axonal regeneration occurs in the CNS following cerebral ischemia (2). During axon sprouting, GAP43 expression increases, thus GAP43 has been used as a marker of axon growth and/or terminal sprouting in stroke models (22). The results of the current study demonstrated high GAP43 protein expression in the HA-treated group compared with that in the 2-VO group, which suggests that sprouting of the injured axon was more active in the HA group following ischemia. Based on the results of $\mathrm{H} \& \mathrm{E}$ staining, it was hypothesized that the neuroprotective effects of HA extracts may include improving axon regeneration following ischemia.

Accompanied with several pathological conditions, including trauma, neuroinflammation and ischemic damage, reactive astrogliosis affects the CNS (18). Reactive astrocytes increase the expression of their structural proteins, such as GFAP and vimentin (23). A previous study has demonstrated that GFAP expression is upregulated in several types of brain injury, including trauma, demyelination and brain ischemia (18). The results of the present study were consistent with these results, demonstrating upregulated GFAP protein expression in the 2-VO group compared with that in the sham-operated group.

A notable role of reactive astrocytes in late-stage neurotrauma is the facilitation of the formation of post-traumatic glial scars and the inhibition of CNS regeneration (18). In particular, they appear to compromise neural graft survival and integration, decreasing the extent of synaptic regeneration, inhibiting neurogenesis in old age and inhibiting the regeneration of severed CNS axons (24). The current study demonstrated that treatment with HA significantly decreased ischemia-reperfusion-induced GFAP expression. Thus, low GFAP expression in the HA-treated group compared with that in the 2-VO group indicated that the HA-treated group demonstrated less inhibition and improved axonal regeneration, which was consistent with the results of LTP and GAP43.

There is a limitation in the present study due to the absence of a sham-operated group receiving HA treatment. However, 
the current study did demonstrate that HA extract reversed the LTP deficit and improved axon regeneration induced by ischemia/reperfusion, which may indicate a potential safe and effective method of using HA in vivo.

Taken together, the results of the present study revealed that HA extracts reversed the LTP deficit and improved axon regeneration induced by ischemia/reperfusion, which may be useful in understanding the molecular mechanism underlying the neuroprotective effects of HA.

\section{Acknowledgements}

Not applicable.

\section{Funding}

The present study was funded by the National Natural Science Foundation of China (grant nos. 81601041 and 81601411), the Medical Foundation of Jieping Wu (grant no. 320.6750.19089-56) and the Youth Incubation Fund of the General Hospital of Tianjin Medical University (grant no. zyyfy2019007).

\section{Availability of data and materials}

All data generated or analyzed during this study are included in this published article.

\section{Authors' contributions}

JX, CW and PX performed the experiments, collected the results and wrote the manuscript. JX and CW analyzed the data and wrote the manuscript. JX and CW confirm the authenticity of all the raw data. All authors read and approved the final manuscript.

\section{Ethics approval and consent to participate}

The current study was approved by the Medical Ethics Committee of Tianjin Medical University General Hospital (Tianjin, China).

\section{Patient consent for publication}

Not applicable.

\section{Competing interests}

The authors declare that they have no competing interests.

\section{References}

1. Mozaffarian D, Benjamin EJ, Go AS, Arnett DK, Blaha MJ, Cushman M, Das SR, de Ferranti S, Després JP, Fullerton HJ, et al; Writing Group Members; American Heart Association Statistics Committee; Stroke Statistics Subcommittee: Heart Disease and Stroke Statistics-2016 Update: A Report From the American Heart Association. Circulation 133: e38-e360, 2016.

2. Wang AR, Hu MZ, Zhang ZL, Zhao ZY, Li YB and Liu B: Fastigial nucleus electrostimulation promotes axonal regeneration after experimental stroke via cAMP/PKA pathway. Neurosci Lett 699: 177-183, 2019.
3. Gondard E, Teves L, Wang L, McKinnon C, Hamani C, Kalia SK, Carlen PL, Tymianski M and Lozano AM: Deep Brain Stimulation Rescues Memory and Synaptic Activity in a Rat Model of Global Ischemia. J Neurosci 39: 2430-2440, 2019.

4. Ueno R, Takase H, Suenaga J, Kishimoto M, Kurihara Y, Takei K, Kawahara N and Yamamoto T: Axonal regeneration and functional recovery driven by endogenous Nogo receptor antagonist LOTUS in a rat model of unilateral pyramidotomy. Exp Neurol 323: 113068, 2020.

5. Ramesh N, Moratti SC and Dias GJ: Hydroxyapatite-polymer biocomposites for bone regeneration: A review of current trends. J Biomed Mater Res B Appl Biomater 106: 2046-2057, 2018.

6. Wei G, Gong C, Hu K, Wang Y and Zhang Y: Biomimetic Hydroxyapatite on Graphene Supports for Biomedical Applications: A Review. Nanomaterials (Basel) 9: 1435, 2019.

7. Huang J, Best SM, Bonfield W, Brooks RA, Rushton N, Jayasinghe SN and Edirisinghe MJ: In vitro assessment of the biological response to nano-sized hydroxyapatite. J Mater Sci Mater Med 15: 441-445, 2004.

8. Liuyun J, Yubao L and Chengdong X: Preparation and biological properties of a novel composite scaffold of nano-hydroxyapatite/chitosan/carboxymethyl cellulose for bone tissue engineering. J Biomed Sci 16: 65, 2009.

9. Farkas E, Luiten PG and Bari F: Permanent, bilateral common carotid artery occlusion in the rat: A model for chronic cerebral hypoperfusion-related neurodegenerative diseases. Brain Res Rev 54: 162-180, 2007.

10. Todd NV, Crockard HA, Russel RW and Picozzi P: Cerebral blood flow in the four-vessel occlusion rat model. Stroke 15: 579, 1984.

11. Yu L, Duan Y, Zhao Z, He W, Xia M, Zhang Q and Cao X: Hydroxysafflor Yellow A (HSYA) Improves Learning and Memory in Cerebral Ischemia Reperfusion-Injured Rats via Recovering Synaptic Plasticity in the Hippocampus. Front Cell Neurosci 12: 371, 2018.

12. Bliss TV and Lomo T: Long-lasting potentiation of synaptic transmission in the dentate area of the anaesthetized rabbit following stimulation of the perforant path. J Physiol 232: 331-356, 1973.

13. Li W, Ye A, Ao L, Zhou L, Yan Y, Hu Y, Fang W and Li Y: Protective Mechanism and Treatment of Neurogenesis in Cerebral Ischemia. Neurochem Res 45: 2258-2277, 2020.

14. Chen MK, Peng CC, Maner RS, Zulkefli ND, Huang SM and Hsieh CL: Geniposide ameliorated fluoxetine-suppressed neurite outgrowth in Neuro2a neuroblastoma cells. Life Sci 226: 1-11, 2019.

15. Wang M, Yao M, Liu J, Takagi N, Yang B, Zhang M, Xu L, Ren J, Fan X and Tian F: Ligusticum chuanxiong exerts neuroprotection by promoting adult neurogenesis and inhibiting inflammation in the hippocampus of ME cerebral ischemia rats. J Ethnopharmacol 249: 112385, 2020.

16. Chen J, Zhang DM, Feng X, Wang J, Qin YY, Zhang T, Huang Q, Sheng R, Chen Z, Li M, et al: TIGAR inhibits ischemia/reperfusion-induced inflammatory response of astrocytes. Neuropharmacology 131: 377-388, 2018.

17. Wang JL and Xu CJ: Astrocytes autophagy in aging and neurodegenerative disorders. Biomed Pharmacother 122: 109691, 2020.

18. Brenner M: Role of GFAP in CNS injuries. Neurosci Lett 565: 7-13, 2014.

19. Lisman J: Glutamatergic synapses are structurally and biochemically complex because of multiple plasticity processes: Long-term potentiation, long-term depression, short-term potentiation and scaling. Philos Trans R Soc Lond B Biol Sci 372: 20160260, 2017.

20. Farhadi Moghadam B and Fereidoni M: Neuroprotective effect of menaquinone-4 (MK-4) on transient global cerebral ischemia/reperfusion injury in rat. PLoS One 15: e229769, 2020.

21. Stokowska A, Atkins AL, Morán J,Pekny T,Bulmer L,Pascoe MC Barnum SR, Wetsel RA, Nilsson JA, Dragunow M, et al: Complement peptide $\mathrm{C} 3$ a stimulates neural plasticity after experimental brain ischaemia. Brain 140: 353-369, 2017.

22. Holahan MR: A Shift from a Pivotal to Supporting Role for the Growth-Associated Protein (GAP-43) in the Coordination of Axonal Structural and Functional Plasticity. Front Cell Neurosci 11: 266, 2017.

23. Erfani S, Moghimi A, Aboutaleb N and Khaksari M: Protective Effects of Nucleobinding-2 After Cerebral Ischemia Via Modulating Bcl-2/Bax Ratio and Reducing Glial Fibrillary Acid Protein Expression. Basic Clin Neurosci 10: 451-459, 2019.

24. Guillamón-Vivancos T, Gómez-Pinedo U and Matías-Guiu J: Astrocytes in neurodegenerative diseases (I): function and molecular description. Neurologia 30: 119-129, 2015. 